\title{
Benefits of group education in IBS
}

Educating IBS patients in a 'school' setting may “...reduce health-care consumption and improve well-being in the long run," report Gisela Ringström and colleagues.

In their randomized controlled study, Ringström et al. compared the effects of providing information on the syndrome in a school setting with written information provided in a guidebook. Patients in the school group attended six $2 \mathrm{~h}$ sessions once a week-in each session, information was provided by a different health-care specialist and time was devoted to group discussion. The effects were assessed by a questionnaire completed at baseline and at 3 months' and 6 months' follow-up.

Perceived patient knowledge and also satisfaction with that knowledge improved significantly more in the school group than in the guidebook group. The school group also had more improvement in the severity of their gastrointestinal symptoms (significant at the 6 month follow-up).

The reduction in the severity of gastrointestinal-specific anxiety was significantly better in the school group.
However, although general anxiety reduced in the school group, there was no difference compared with the guidebook group; the authors suggest this is not surprising given the school group's focus on gastrointestinal symptoms. Several aspects of health-related quality of life also improved in the school group, but compared with the guidebook group these changes were mostly nonsignificant.

Ringström et al. believe that written information may work for IBS patients in primary care who have mild symptoms, but conclude that "...management in a group setting could be useful for patients in secondary/tertiary care with more severe symptoms." To minimize costs, the authors are studying the effectiveness of nurse-led education in three $2 \mathrm{~h}$ sessions.

Natalie J. Wood

Original article Ringström, G. et al. Structured patient education is superior to written information in the management of irritable bowel syndrome: a randomized controlled study. Eur. J. Gastroenterol. Hepatol. 22, 420-428 (2010) 